\title{
Control Strategy of Constant-Speed Air-Conditioners Based on Virtual State of Charging Priority
}

\author{
Yanbo Che $\mathbb{D}^{1},{ }^{1}$ Zheng Li, $^{1}$ Wei He, ${ }^{2}$ Yuancheng Zhao ${ }^{D},{ }^{1}$ and Ruiping Zhang ${ }^{3}$ \\ ${ }^{1}$ Key Laboratory of Smart Grid of Ministry of Education, Tianjin University, Tianjin 300072, China \\ ${ }^{2}$ State Grid Jiangxi Electric Power Research Institute, Nanchang 330096, China \\ ${ }^{3}$ College of Automation and Electrical Engineering of Lanzhou Jiaotong University, Gansu 730070, China
}

Correspondence should be addressed to Yanbo Che; lab538@163.com

Received 21 March 2019; Revised 18 May 2019; Accepted 12 June 2019; Published 1 July 2019

Academic Editor: Federica Caselli

Copyright (C) 2019 Yanbo Che et al. This is an open access article distributed under the Creative Commons Attribution License, which permits unrestricted use, distribution, and reproduction in any medium, provided the original work is properly cited.

\begin{abstract}
As typical thermostatically controlled loads (TCL) driven by constant-speed compressor, constant-speed air-conditioners play important roles in demand-side response for their abilities of energy conversion and storage. Their great potential for load regulation can be incorporated into power system scheduling through demand response. In view of their operating characteristics, a virtual energy storage (VES) model of thermostatically controlled loads with electrical and thermal parameters is established. This model is discretized and linearized to simplify calculation. By analyzing the control function and constraints of the VES model, the control strategy of VES of constant-speed air-conditioners load with virtual charging state priority is proposed. Example analysis shows that this strategy can solve and alleviate power shortage problem of the system by participating in demand response, which provides methodological support for constant-speed compressor temperature-control load to participate in the system operation.
\end{abstract}

\section{Introduction}

With the development of advanced measurement techniques [1], power system stakeholders are becoming pluralistic, the interaction between power generation and load response is becoming more visible, and with emerging technologies, it is easy to resolve the problem of energy shortage [2]. Demand response plays a key role in power management; once the load curve is derived, it is easy to track the power shortage, shave the peak, and fill the valley by defining user attractive schemes. The implementation of internet + smart energy strategies [3] has a great significance in relation to sale electricity reform and the construction of smart cities. Demand response can be varied by price signals and incentive measures. To control demand response by price signals, demand elasticity is used to encourage users to adjust their load in accordance with price changes. Demand response is controlled by the incentive measures; relevant policies are used to encourage users to transfer control rights through corresponding policies, so as to achieve the goal of minimum cost.
Air-conditioner load operation is characterized as short time, large proportion, and high peak value. Without the improvement, it will increase the peaking pressure of the power grid and lead to imbalance of supply and demand. Allowing resident users to transfer control of air conditioning resources is the most efficient way to solve the problem of power shortage and energy consumption through, while modeling air-conditioning resources is the key to fully exploit the scheduling margin and effectively implement direct load control of demand response [4].

At present, two modeling methods for TCLs are commonly accepted: equivalent thermal parameter (ETP) based on circuit simulation $[5,6]$ and calculation based on cold and heat load [7]. Reference [8] proposed state sequence algorithm to suppress the energy fluctuation of the tie-line by controlling the start-stop state of the thermostatically controlled loads. The specific characteristics of household load are fully considered and then a load model, taking account of users' behavior, is established in [9]. Reference [10] uses a thermal comfort model to estimate a residential comfort level and build a dispatching system for large-scale 


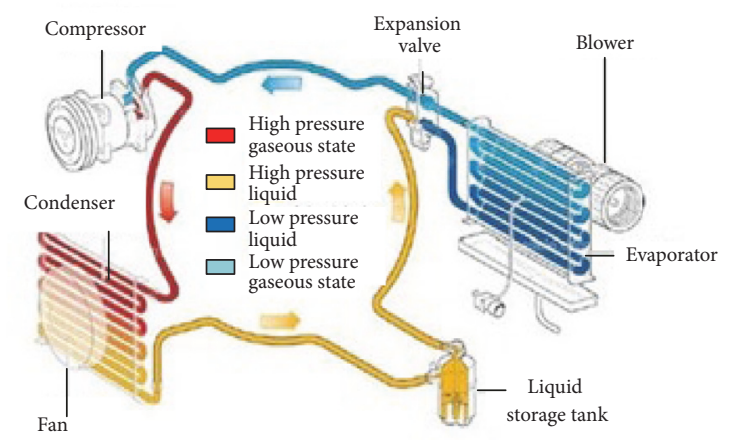

FIGURE 1: Schematic diagram of constant-speed air-conditioner.

TCL-based load. Reference [11] establishes a thermodynamic model of air-conditioner load based on the double-coupled partial differential equation and proposes the concept of VES. Reference [12] compares three control methods, (i) including air-conditioner load (ii) changing compressor start-stop valve setting and (iii) duty cycle control. But it lacks quantitative evaluation of their corresponding capacity.

By guaranteeing the basic comfort requirements of users $[13,14]$, air-conditioning can be equivalent to VES devices. Reference [15] establishes a VES model based on airconditioner load thermodynamic model and human thermal comfort model, which proves the potential and feasibility of applying air-conditioner load VES on demand response. Reference [16] proposed an optimal allocation model of energy-efficient power plants considering user comfort level. Its logic provides solid theoretical support for the deep participation of thermostatically controlled loads in demandside response. Reference [17] presents a VES model of electric heating load and proposes a control strategy of electric heating load based on virtual state of charge priority table, which provides good technical support for this paper.

This paper presents analysis of constant-speed airconditioning. A VES model based on first-order thermodynamic model is proposed, which reflects the load electrothermal conversion of constant-speed air-conditioning and fulfill the potential of demand-side response. Based on the VES model, a VSOC prior to VES control strategy for constantspeed air-conditioning load is established. Simulation results validate the proposed control strategy of constant-speed airconditioning in demand-side response and can play a major role in power management to balance the load curve.

\section{VES Model of Constant-Speed Air-Conditioning}

With the rapid increase in electricity demand, an energystored constant-speed air-conditioning becomes a load with great potential to vary a demand response [18] according to proposed strategy. By adopting reasonable control methods to simulate demand response, constant-speed airconditioners can quickly respond to grid side dispatch, reduce load demand during the peak hours, and alleviate the shortage of power supply to some extent. Compared to investment on expanding the capacity of power plants, cost on demand-side response controlling and the influence of participants on reasonable comfort level is lower [19].

2.1. Operational Characteristics. Constant-speed airconditioner mainly consists of the refrigeration system [20], the ventilation system, and the electrical system. Its core operating principle is "inverse Carnot cycle"; detailed processes are shown in Figure 1.

The constant-speed air-conditioner usually adopts compressor driven by single-phase asynchronous motor, which means the speed in stable operation is determined by grid frequency. In order to maintain a specific indoor temperature, the compressor needs to be continuously on and off, which is called start-stop regulation.

Referring to experimental results in [21], constant-speed air-conditioner is a constant power load within the allowable threshold range of power grid operation. In terms of dynamic characteristics, voltage flicker and frequency fluctuation of power grid will affect the processes of starting the cooling, constant temperature operation, and shutdown of airconditioner directly, which will lead to dissatisfaction of users' comfort level and damage of air-conditioner. Frequent start-up and shutdown of constant-speed air-conditioner should be avoided because it will increase the wear of moving parts of air-conditioner machinery and bring greater peak impact and energy loss to power grid.

2.2. Equivalent Thermal Parameter Model. Based on the ETP modeling method [15], this paper establishes an equivalent thermal parameter model for room thermal energy conversion, which hereinafter referred to as the ETP model.

Considering the differences among solid temperature, gas temperature, heat capacity, and thermal resistance of exterior and interior walls, the classical third-order state-space thermodynamic model comprehensively analyzes the energy transfer process between the environment and the room. Neglecting the difference between inner and outer walls, the widely used second-order thermodynamic model [22] can be obtained by considering the practical application and calculating speed. When the temperature changes smoothly, there is no obvious difference between the temperature of gas 


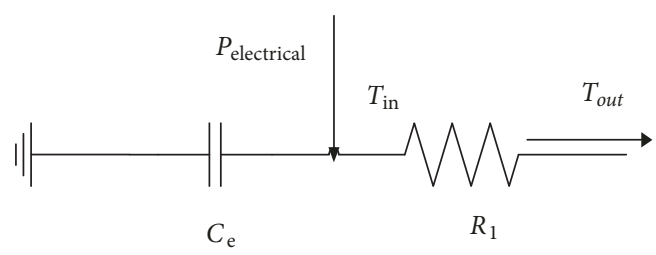

FIgURE 2: First-order ETP model.

and solid in the room. In order to enhance the practicability of the model, the second-order ETP model can be reduced to the first-order ETP model, as shown in Figure 2, where $P_{\text {electrical }}$ represents the refrigeration or heating rate, KW.

Except for special instructions, this paper takes heating as an example, so hereinafter referred to as heating rate. $T_{\text {in }}$ and $T_{\text {out }}$ denote the temperature in and outside of room in ${ }^{\circ} \mathrm{C}$, respectively. $C_{\mathrm{a}}$ represents the heat capacity of the gas $\mathrm{J} /{ }^{\circ} \mathrm{C} ; R_{1}$ represents the heat transfer resistance of the energy between the room and the external environment ${ }^{\circ} \mathrm{C} / \mathrm{W} . \mathrm{C}_{\mathrm{e}}$ denotes the equivalent heat capacity in the room. According to Kirchhoff's theorem, the state space equation of the firstorder ETP model is

$$
\dot{T}_{\text {in }}=\frac{1}{R_{1} C_{\mathrm{e}}}\left(T_{\text {out }}-T_{\text {in }}\right)+\frac{P_{\text {electrical }}}{C_{\mathrm{e}}}
$$

$T_{\text {in }}$ is the rate of temperature change. Formula (1) can be transformed into

$$
\frac{T_{\text {in }}-T_{\text {out }}}{R_{1}}+C_{\mathrm{e}} \frac{d T_{\text {in }}}{d \mathrm{t}}=P_{\text {electrical }}
$$

The physical meaning of each part of (2) is analyzed as follows: $\left(T_{\text {in }}-T_{\text {out }}\right) / R_{1}$ indicates the heat loss caused by the temperature difference between the room and the external environment, $R_{1}$ reacts to the extent to which heat loss is hindered, $C_{\mathrm{e}} d T_{\text {in }} / d \mathrm{t}$ represents the thermal power required for room heating, and $C_{\mathrm{e}}$ represents the amount of energy required for temperature changes.

\subsection{VES Model}

2.3.1. Thermal Parameter Section. The response potential of demand-side resources mainly depends on the response time interval. Considering the different response time of demand-side resources, the virtual thermal storage parameter model in Figure 3 can be obtained by equivalently changing Figure 2.

In Figure 3, $T_{\text {in }}{ }^{t}$ represents the indoor temperature at time $t$ in ${ }^{\circ} \mathrm{C} ; S$ represents the switch state, with 0 disconnected and 1 controlled. The action from the closed state to disconnected or controlled state can be regarded as the beginning of the discharging stage, and the reverse action can be regarded as the beginning of the charging stage.

$P_{\text {electrical }}$ represents the external charging power supplied in VES and it depends on the type of constant-speed airconditioner. $C_{\mathrm{e}}$ represents the core part of energy storage in the VES thermal parameter model, mainly used in changing room temperature and its energy storage capacity mainly

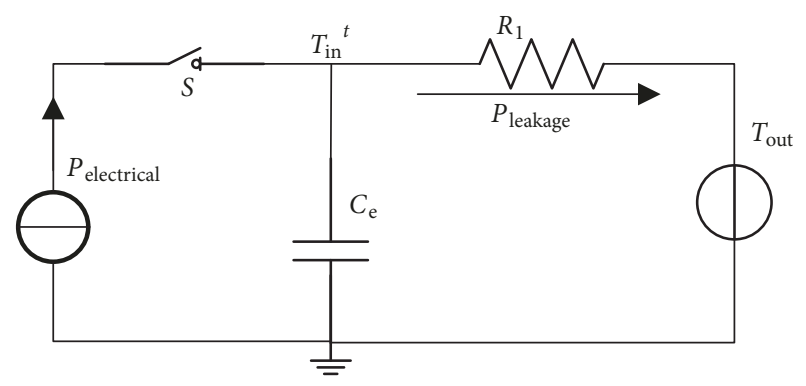

FIGURE 3: Thermal parameters part of the VES model.

depends on the temperature limit of the TCL and the current temperature. $P_{\text {leakage }}=\left(T_{\text {in }}{ }^{t}-T_{\text {out }}\right) / R_{1}$ represents the leakage current in the virtual thermal storage parameter model, mainly depending on the energy loss caused by temperature difference.

The power before the transfer of VES control is set as the base power $P_{\text {base }}$. When the TCL participating in the demand response, $P_{\text {electrical }}$, increases, the VES can be considered to be in charging state and vice versa in discharging state. Charging and discharging power can be expressed as

$$
\begin{gathered}
P_{\text {disc }}=S(t-1)[1-S(t)]\left(P_{\text {electrical }}-P_{\text {base }}\right) \\
P_{\text {char }}=S(t)[1-S(t-1)]\left(P_{\text {electrical }}-P_{\text {base }}\right)
\end{gathered}
$$

$P_{\text {char }}$ is charging power and $P_{\text {disc }}$ is discharging power. $S(t)$ represents the switching state at $t$ time. The charging and discharging capacity of VES is derived from the change of state rather than the persistence of state like that of batteries.

Let $T_{\text {in }}\left(t_{0}\right)=\mathrm{C}$, and from formula (2), we have

$$
\begin{aligned}
T_{\text {in }}(t)= & T_{\text {out }}(t)+S R_{1} P_{\text {electrical }}(t) \\
& -\left(T_{\text {out }}(t)+S R_{1} P_{\text {electrical }}(t)-C\right) \mathrm{e}^{-t / R_{1} C_{\mathrm{e}}}
\end{aligned}
$$

From formula (4), the charging and discharging time is

$$
t_{\text {on } / \text { off }}=R_{1} C_{\mathrm{e}} \ln \left(\frac{C-T_{\text {out }}(t)-S R_{1} P_{\text {electrical }}(t)}{T_{\text {in }}(t)-T_{\text {out }}(t)-S R_{1} P_{\text {electrical }}(t)}\right)
$$

In addition to the inherent room parameters, the charging and discharging time depend on internal and external temperatures, the state of switch function, and electric power. The energy of VES load is stored in capacitor $C_{e}$, and by using the relationship between temperature and power in the ETP model, the maximum charge capacity of VES is

$$
Q_{\text {capacity }}=C_{\mathrm{e}}\left(T_{\max }-T_{\text {min }}\right)
$$

Based on the principle of energy conservation, the charge capacity at time $t$ is

$$
Q(t)=Q\left(t_{0}\right)+\int_{t_{0}}^{t} P_{C_{\mathrm{e}}}(\xi) d \xi
$$

where $P_{C e}(t)$ represents the power flowing into the capacitor $C_{\mathrm{e}}$. 


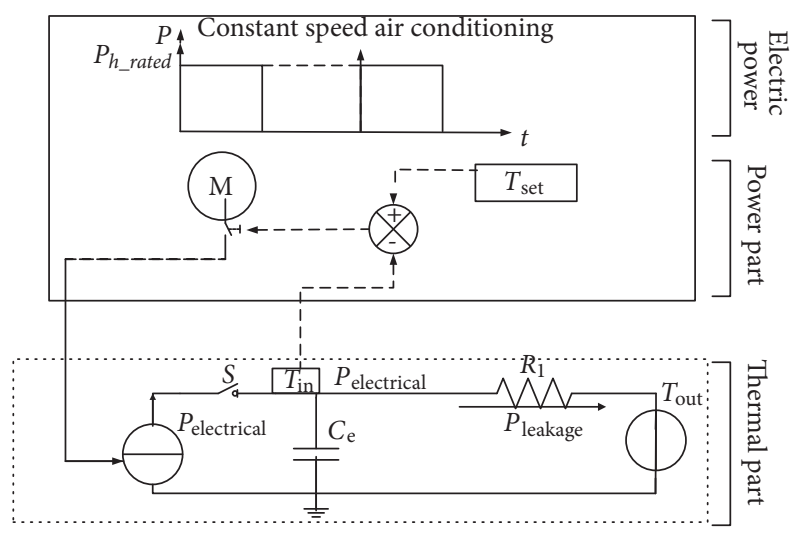

FIGURE 4: VES model.

Substituting (4) into the above formula, we have

$$
\begin{aligned}
& Q(t)=C_{\mathrm{e}} T_{\text {in }}\left(t_{0}\right)+C_{\mathrm{e}} \int_{t_{0}}^{t} T_{\text {in }}(\xi) d \xi=C_{\mathrm{e}} T_{\text {in }}\left(t_{0}\right) \\
& \quad+C_{\mathrm{e}} \int_{t_{0}}^{t}\left(T_{\text {out }}(t)+S R_{1} P_{\text {electrical }}(t)\right. \\
& \left.\quad-\left(T_{\text {out }}(t)+S R_{1} P_{\text {electrical }}(t)-\mathrm{C}\right) \mathrm{e}^{-t / R_{1} C_{\mathrm{e}}}\right) d \xi
\end{aligned}
$$

The United States Advanced Battery Consortium (USABC) defines State of Charge (SOC) in "electric vehicle battery laboratory manual" as the ratio of the remaining battery capacity to the rated capacity under the same conditions at a certain discharge rate. Following its definition, Virtual State of Charge (VSOC) is proposed as the ratio of residual energy to rated capacity under the same charging and discharging power. Therefore, VSOC represents the response capability of VES at the current stage.

$$
\mathrm{VSOC}=\frac{Q(t)}{Q_{\text {capacity }}}
$$

2.3.2. Electrical Parameter Part. Assume that the external power grid is stable; the constant-speed air-conditioner works at a fixed speed and controls room temperature by starting and stopping the compressor. Fully considering the startup characteristics, electric power can be expressed as

$$
P_{\text {electrical }}= \begin{cases}\eta \mu S_{\mathrm{Fac}}(t) \cdot P_{\text {Fac_rated }} \delta(t) & t \leq \varepsilon \\ \eta S_{\mathrm{Fac}}(t) \cdot P_{\text {Fac_rated }} & t>\varepsilon\end{cases}
$$

where $\eta$ denotes the energy efficiency ratio, which depends on the type of air-conditioner; $\mu$ denotes the impact power multiple, which mainly depends on the parameters of airconditioning; $P_{\text {Fac_rated }}$ denotes the rated power in $\mathrm{KW} ; \delta(t)$ denotes the impact at a certain time as a unit impulse function; $\varepsilon$ denotes the value approaching $0, \lim (\varepsilon)=0$; and $S_{\mathrm{Fac}}(t)$ is like $S_{\mathrm{h}}(t)$, which indicates the switching state, but it needs to distinguish refrigeration and heating conditions.

In conclusion, the structure of the VES model is shown in Figure 4. Solid lines with arrows indicate the direction of energy flow, and dashed lines with arrows indicate the direction of signal flow.

\section{Preprocessing Based on Policy Model}

Advanced measurement system [23] records data such as user power consumption at a specific time interval and can transmit data to the data center system through the communication network in a specific time period, which provides a hardware basis for demand response.

The communication capacity of advanced measurement systems supporting demand response is limited. In order to prevent communication data congestion and improve communication timeliness, it is necessary to discretize and linearize the original VES model.

3.1. Operational characteristics. In the process of demand response, constant-speed air-conditioner is intermittent, and $P_{\text {electrical }}$ is the discrete variable. $\mathrm{T}_{\text {in }}(t)$, as a continuous variable, has relatively larger demand of traffic flow. Formula (4) cannot describe $\mathrm{T}_{\text {in }}(t)$ in actual data transmission. Therefore, it is necessary to discretize the VES model.

Assuming the time step is $\Delta t$, the solution of (2) can be obtained

$$
\begin{aligned}
T_{\text {in }}{ }^{t+1}= & T_{\text {out }}{ }^{t+1}+S R_{1} P_{\text {electrical }} \\
& -\left(T_{\text {out }}{ }^{t+1}+S R_{1} P_{\text {electrical }}-T_{\text {in }}{ }^{t}\right) \mathrm{e}^{-\Delta t / R_{1} C_{\mathrm{e}}}
\end{aligned}
$$

where $T_{\text {in }}{ }^{t}$ denotes the temperature inside the room at time $t$ in ${ }^{\circ} \mathrm{C}$; $T_{\text {in }}{ }^{t+1}$ denotes the temperature inside the room at time $t+1$ in ${ }^{\circ} \mathrm{C} ; T_{\text {out }}{ }^{t}$ denotes the temperature outside the room at time $t$ in ${ }^{\circ} \mathrm{C} ; T_{\text {out }}{ }^{t+1}$ denotes the temperature outside the room at time $t+1$ in ${ }^{\circ} \mathrm{C}$; and $S$ denotes that the switch function is 0 or 1 according to the operating conditions. $\Delta t$ denotes the communication time interval.

3.2. Model Linearization. Temperature change is a slow process. When the response time of participating demand side is shorter, the external environment is considered as no change, i.e., $T_{\text {out }}{ }^{t}=T_{\text {out }}{ }^{t+1}$. Setting the room temperature range to [ $\left.T_{\min }, T_{\max }\right]$, control cycle of $P_{\text {electrical }}$ to $t_{\mathrm{cyc}}$, where the time with electric power input is $\mathrm{t}_{\mathrm{on}}$, and the time without electric power input is $t_{\text {off. }}$. From the iterative calculation of $T_{\min }$ and $T_{\max }$ with the formula (11)

$$
\begin{aligned}
T_{\max }= & \left(T_{\text {out }}+R_{1} P_{\text {electrical }}\right)\left(1-\mathrm{e}^{-t_{\text {on }} \Delta t / R_{1} C_{\mathrm{e}}}\right) \\
& +T_{\min } \mathrm{e}^{-t_{\text {on }} \Delta t / R_{1} C_{\mathrm{e}}} \\
T_{\min }= & T_{\text {out }}\left(1-\mathrm{e}^{-t_{\text {of }} \Delta t / R_{1} C_{\mathrm{e}}}\right)+T_{\max } \mathrm{e}^{-t_{\text {off }} \Delta t / R_{1} C_{\mathrm{e}}} \\
t_{\text {cyc }}= & t_{\text {on }}+t_{\text {off }}
\end{aligned}
$$

Solve (12) to obtain the charging and discharging time as

$$
\begin{aligned}
& t_{\text {off }}=\frac{R_{1} C_{\mathrm{e}}}{\Delta t} \ln \left(\frac{T_{\text {max }}-T_{\text {out }}}{T_{\text {min }}-T_{\text {out }}}\right) \\
& t_{\text {on }}=\frac{R_{1} C_{\mathrm{e}}}{\Delta t} \ln \left(\frac{T_{\text {min }}-T_{\text {out }}-R_{1} P_{\text {electrical }}}{T_{\text {max }}-T_{\text {out }}-R_{1} P_{\text {electrical }}}\right)
\end{aligned}
$$




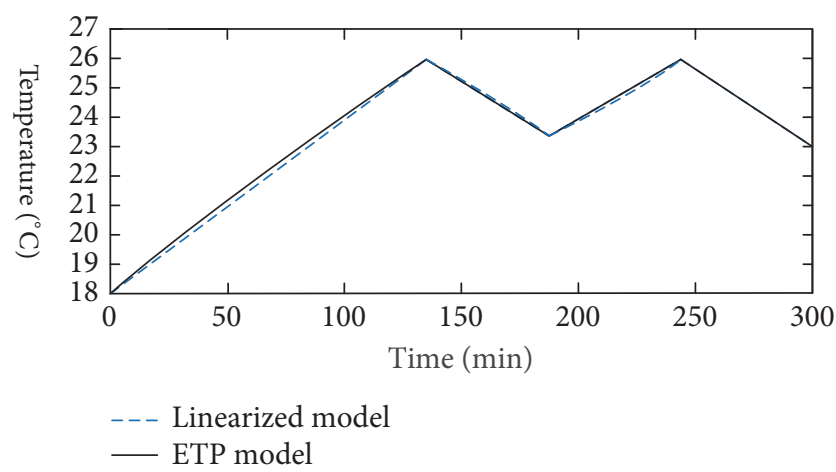

FIGURE 5: Comparison of temperature curves between linearized model and original ETP model.

With the ratio of time interval $\Delta t$ to total switching time $t_{o n}$ and $t_{\text {off }}$, the temperature change of each iteration is described as

$$
\begin{array}{ll}
T_{\text {in }}{ }^{t+1}=T_{\text {in }}{ }^{t}+\frac{\Delta t}{t_{\text {on }}}\left(T_{\text {max }}-T_{\text {min }}\right) & s=1 \\
T_{\text {in }}{ }^{t+1}=T_{\text {in }}{ }^{t}-\frac{\Delta t}{t_{\text {off }}}\left(T_{\text {max }}-T_{\text {min }}\right) & \mathrm{s}=0
\end{array}
$$

taking the air-conditioner heating as an example to verify the rationality of linearization. The simulation parameters are $R_{1}=0.1208^{\circ} \mathrm{C} / \mathrm{W} ; C_{\mathrm{e}}=3599.3 \mathrm{~J} /{ }^{\circ} \mathrm{C}[24] ; P_{\text {electrical }}=400 \mathrm{~W} ; T_{\text {min }}$ $=23^{\circ} \mathrm{C} ; T_{\max }=26^{\circ} \mathrm{C} ; \Delta t=1 \mathrm{~min} ; T_{\text {out }} \equiv 0^{\circ} \mathrm{C}$; and $T_{\text {in }}{ }^{\circ}=18^{\circ} \mathrm{C}$, and the simulation results of MATLAB platform are shown in Figure 5. The solid line represents the temperature changes with time from the first-order ETP model and the dotted line represents the result shown in the linearized ETP model. By conducting comparative analysis of the temperature rise process from $18^{\circ} \mathrm{C}$ to $26^{\circ} \mathrm{C}$ and the heat preservation process from $23^{\circ} \mathrm{C}$ to $26^{\circ} \mathrm{C}$, the results show that the linearization effect is better in the shorter temperature range and the part near the temperature limit is better.

The correlation coefficients $R$ for the two curves are calculated as

$$
R(X, Y)=\frac{\operatorname{Cov}(X, Y)}{\sqrt{D(X) \cdot D(Y)}}
$$

where $R(X, Y)$ denotes the correlation coefficient of $X$ and $Y$; the closer the correlation coefficient is to 1 , the greater the correlation between the two curves is, and the less the influence of substitution is; $\operatorname{Cov}(X, Y)$ denotes the covariance of $X$ and $Y ; D(X), D(Y)$ denotes the standard deviation of $X$ and $Y$.

Within 300 minutes, the overall correlation coefficient is $R=0.99856$ between the linearized ETP model and the first-order ETP model. Generally speaking, the VES load below the minimum temperature does not participate in the demand response. The correlation coefficient of the heating process part and the normal working part is $R=0.99919$. It is considered that the linearization processing has a good fitting effect with the original model and will not have any greater impact on the relevant results.

\section{VSOC Priority VES Control Strategy}

VSOC prioritized VES control strategy is to select the one with higher VSOC to participate in demand response for the same type of VES equipment.

4.1. Control Model of Constant-Speed Air-Conditioning. Community users are involved in demand response. Assuming that the external environment does not change during the response period, the energy consumption only occurs when the room temperature and the external temperature change. Communication data refresh interval is $\Delta t$.

The change of the switch determines the working state of the VES model, and the switch function must highlight the state changes at different times. Setting switch function $S_{\mathrm{Fac}}=1$ as charging and $S_{\mathrm{Fac}}=0$ as discharging, then the VES of a constant-speed air-conditioner at a certain time $t$ is

$$
\begin{aligned}
& P^{j}{ }_{\text {Fac_disc }}(t)=S_{\mathrm{Fac}}^{j}(t-1)\left[1-S_{\mathrm{Fac}}^{j}(t)\right] P^{j}{ }_{\text {Fac_rated }} \\
& P^{j} \text { Fac_char }(t) \\
& = \begin{cases}\mu S_{\mathrm{Fac}}^{j}(t)\left[1-S_{\mathrm{Fac}}^{j}(t-1)\right] \cdot P^{j}{ }_{\text {Fac_rated }} \delta(t) & t \leq \varepsilon \\
S_{\mathrm{Fac}}^{j}(t)\left[1-S_{\mathrm{Fac}}^{j}(t-1)\right] \cdot P^{j}{ }_{\text {Fac_rated }} & t>\varepsilon\end{cases}
\end{aligned}
$$

Subscripts Fac_disc and Fac_char denote discharging power and charging power of constant-speed air-conditioner, respectively. Superscript $j$ denotes the $j$-th VES unit, and $P^{j}{ }_{\text {Fac_rated }}$ denotes rated power of the $j$ th constant-speed airconditioner. $\mu$ is the multiple of impact power, and $\delta(t)$ is a unit impulse function.

The charging process of constant-speed air-conditioner VES is divided into two stages. At the beginning, an impact power with $\mu$ times of rated power lasts a very short time period, which then quickly falls to the rated power. Due to the impact power, it is necessary to limit the number of times in relation to start-stop loads per unit of time and start-stops during a single-load control period strictly.

At a certain moment $t$, VSOC $^{j}$ Fac of the $j$ th constantspeed air-conditioner or virtual energy storage unit is

$$
\begin{aligned}
\operatorname{VSOC}_{\mathrm{Fac}}^{j}(t) & =\frac{Q_{\mathrm{Fac}}(t)}{Q_{\text {Fac_capacity }}} \\
& =\frac{C_{\mathrm{e}}^{j}\left(T^{j}{ }_{\text {Fac }}(t)-T^{j}{ }_{\text {Fac_min }}\right)}{C_{\mathrm{e}}^{j}\left(T^{j}{ }_{\text {Fac_max }}-T^{j}{ }_{\text {Fac_min }}\right)} \\
& =\frac{T_{\text {Fac }}^{j}(t)-T^{j}{ }_{\text {Fac_min }}}{T^{j}{ }_{\text {Fac_max }}-T^{j}{ }_{\text {Fac_min }}}
\end{aligned}
$$

At a certain moment $\mathrm{t}$, the $j$ th constant-speed airconditioner VES VSOC ${ }^{j}$ Fac is

$$
\begin{aligned}
\operatorname{VSOC}_{\text {Fac }}^{j}(t+1) & \\
= & \frac{\Delta t-\sum_{0}^{i} \varepsilon_{j}}{S^{j}{ }_{\text {Fac }}(t) t^{j}{ }_{\text {Fac_on_max }}-\left(1-S^{j}{ }_{\text {Fac }}(t)\right) t^{j}{ }_{\text {Fac_off_max }}} \\
& +\operatorname{VSOC}_{\text {Fac }}^{j}(t)
\end{aligned}
$$




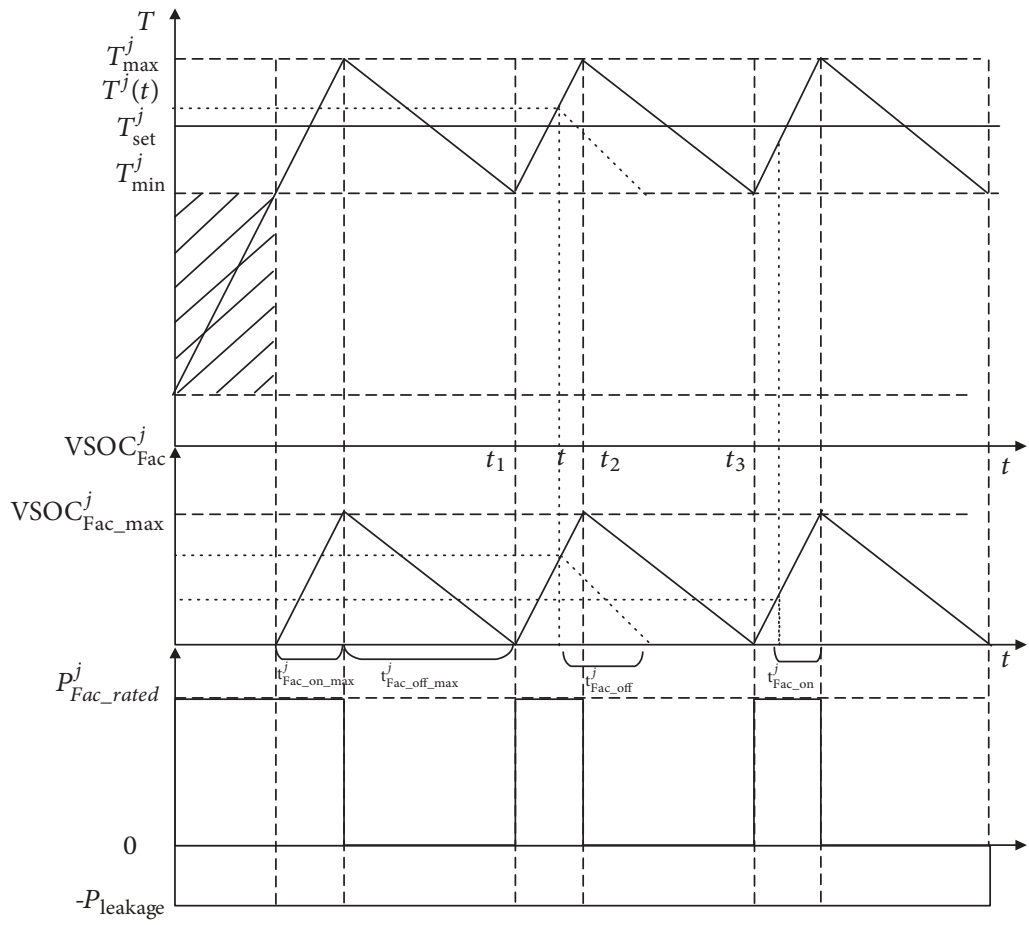

FIGURE 6: Relation diagram of linearized temperature curve, VSOC curve, and thermal power.

where $i$ represents the $i$-th constant-speed air-conditioner in the start-up sequence, $t_{\mathrm{i}}$ represents the time point of the $i$-th start-up, and k represents the number of constant-speed airconditioners needed to start-up during the time interval $\Delta t$.

To reduce the impact power of constant-speed airconditioning in demand response and prevent the maloperation of relay protection device due to the superposition of power peaks caused by start-up, two constraints are added to (19).

$$
\begin{aligned}
& \frac{t^{j}{ }_{\text {Fac_off }}}{t^{j}{ }_{\text {Fac_off_max }}}=\frac{\text { VSOC }_{F a c}^{j}(t)-V_{\text {VSOC }}^{j}}{\text { VSOC_min }_{\text {Fac_max }}^{j}-V_{\text {VSOC }}^{j}} \\
& \frac{1-t_{\text {Fac_min }}^{j}{ }_{\text {Fac_on }}}{t^{j}{ }_{\text {Fac_on_max }}}=\frac{\text { VSOC }_{F a c}^{j}(t)-V_{\text {VSOC }}^{j}}{\text { VSOC_min }_{\text {Fac_max }}^{j}-V_{\text {VSOC }}^{j}}
\end{aligned}
$$

The first one indicates that the interval between start times of every two air-conditioners should be greater than $\varepsilon_{\mathrm{i}}$ during the demand response process; the second one indicates the restriction to duration that the air-conditioner started within the time interval bearing the impact power.

Figure 6 represents the relationships among linearized temperature curve, VSOC curve, and electric power and thermal power. The shaded part indicates that the VES equipment not reaching the steady-state temperature is not considered. For the $j$ th VES of air-conditioner at time $t$, $T^{j}(\mathrm{t})$ denotes the temperature; $t^{j}{ }_{\text {Fac_off }}$ denotes the remaining discharge time; $t^{j}{ }_{\text {Fac_off_max }}$ denotes the maximum discharge time; $t^{j}$ Fac_on_max denotes the maximum charge time; and $t^{j}{ }_{\text {Fac_on }}$ denotes the remaining charge time.
Using triangle similarity principle, the virtual charge state of VES in constant-speed air-conditioner can be expressed by the ratio of the difference between the current temperature and limit value. The essence of discretization and linearization of the VES model is to describe electrical parameters with thermal parameters, i.e., to complete the conversion of electrical parameters to thermal parameters in the time domain.

Charging and discharging time is an important constraint index. On the one hand, it can prevent the VES unit of airconditioner from overcharged or overdischarged maintaining the temperature fluctuation of air-conditioner within the set range. On the other hand, it can keep the state of equipment during communication time interval to avoid affecting the control accuracy and bringing unnecessary network side fluctuation.

$$
\begin{aligned}
& \frac{t^{j}{ }_{\text {Fac_off }}}{t^{j}{ }_{\text {Fac_off_max }}}=\frac{\text { VSOC }_{F \text { ac }}^{j}(t)-V_{\text {VSOC }}^{j}}{\text { VSOC_min }_{\text {Fac_max }}^{j}-V_{\text {VSOC }}^{j}} \\
& \frac{1-t_{\text {Fac_min }}^{j} \text { Fac_on }}{t^{j}{ }_{\text {Fac_on_max }}}=\frac{\text { VSOC }_{F \text { ac }}^{j}(t)-\text { VSOC }_{\text {Fac_min }}^{j}}{\text { VSOC }_{\text {Fac_max }}^{j}-\text { VSOC }_{\text {Fac_min }}^{j}}
\end{aligned}
$$

Further simplification is available:

$$
\begin{aligned}
& t_{\text {Fac_off }}^{j}=\operatorname{VSOC}_{\text {Fac }}^{j}(t) t_{\text {Fac_off_max }}^{j} \\
& t_{\text {Fac_on }}^{j}=\left(1-\operatorname{VSOC}_{\text {Fac }}^{j}(t)\right) t_{\text {Fac_on_max }}^{j}
\end{aligned}
$$




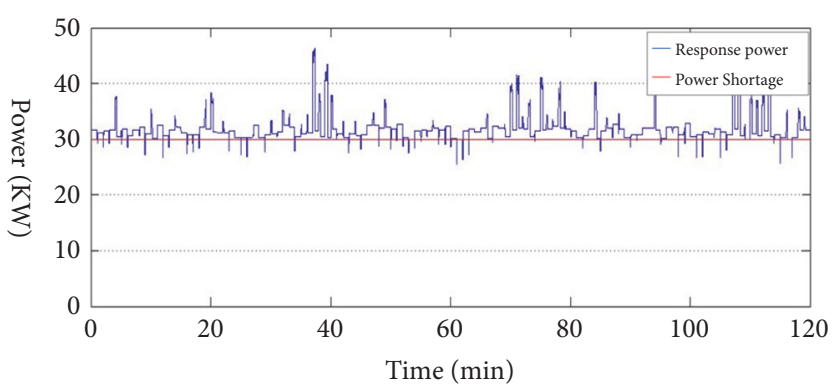

(a) Response power change diagram

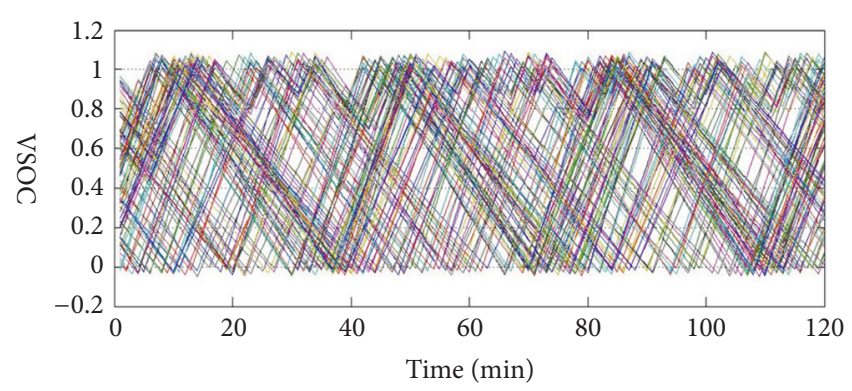

(b) VSOC change chart of 100 constant-speed air-conditioner with VES

FIGURE 7: Power change and VSOC change chart in 120 minutes after optimization.

To sum up, taking discharge as an example, the control model of VES for constant-speed air-conditioning is established.

$$
\begin{aligned}
& P_{\text {Fac_disc }}^{j}(t)=S_{\mathrm{Fac}}^{j}(t-1)\left[1-S_{\mathrm{Fac}}^{j}(t)\right] P_{\text {Fac_rated }}^{j} \\
& \text { VSOC }_{\text {Fac }}^{j}(t+1) \\
& =\frac{\Delta t-\sum_{0}^{i} \varepsilon_{j}}{S_{\text {Fac }}^{j}(t) t^{j}{ }_{\text {Fac_on_max }}-\left(1-S^{j}{ }_{\text {Fac }}(t)\right) t^{j}{ }_{\text {Fac_off_max }}} \\
& + \text { VSOC }_{\mathrm{Fac}}^{j}(t) \\
& t_{\text {Fac_off }}^{j}=\text { VSOC }_{\text {Fac }}^{j}(t) t_{\text {Fac_off_max }}^{j}
\end{aligned}
$$

4.2. Control Strategy and Algorithms Analysis. The main idea of the control strategy for constant-speed air-conditioners is to control the on-off state of VES in the light of their VSOCs. The VES equipment with higher VSOC will be turned off in priority. The VSOC value is in the range of $0-1$. Its main objective function is to satisfy the power vacancy within the communication time interval, and the marginal limitation is the discharge time is longer than the communication time interval. In addition, it is necessary to satisfy the relevant constraints in the control model and ensure that the power peak is as small as possible. Specific control functions are shown as formulas.

$$
\begin{aligned}
P_{\mathrm{s}}{ }^{t} & \leq \min \sum_{\mathrm{A}} P_{\text {Fac_disc }}^{t}\left(j_{n}\right) \\
P_{\mathrm{s}}^{t}-\sum_{\mathrm{A}} P_{\text {Fac_disc }}^{t}\left(j_{n}\right) & \leq \min \sum_{\mathrm{C}_{\mathrm{Q}} \mathrm{A}} P_{\text {Fac_disc }}^{t}\left(j_{n}\right) \\
0 & \leq \operatorname{VSOC}_{\mathrm{Fac}}^{t}\left(j_{n}\right) \leq 1 \\
t^{j_{n}}{ }_{\text {Fac_off }} & \geq \Delta t \\
t^{j_{n}}{ }_{i+1}-t^{j_{n}}{ }_{i} & \geq \varepsilon_{j_{n}} \\
k & <\frac{\Delta t}{\min _{i \in[0, k]} \varepsilon_{i}}
\end{aligned}
$$

Among them, $P_{\mathrm{s}}^{\mathrm{t}}$ represents the power vacancy at time $t$; $Q$ is the set in which air-conditioners are arranged according to their VSOCs from large to small; $j_{n}$ is the element of $Q$ sets, and $n$ represents the position of $\mathrm{j}$ in the new set, $A$ denotes the controlled set arranged from large to small according to VSOC; and $C_{Q} A$ denotes the complement set of $A$ whose complete set is $Q$. The new restriction condition expresses the priority of controlled virtual energy storage discharge and then supplements the power vacancy based on the size of VSOC.

Using MATLAB as the simulation platform, major program implementation steps include refreshing data status, dealing with VSOC overlimit problem, generating control queue $Q$ based on VSOCs, calculating whether the power vacancy is met, generating startup queue based on impact power, processing related startup queue, and iteratively updating VES status.

\section{Example Analysis}

5.1. Algorithmic Case. The parameters of the example are as follows. There are 100 constant-speed air-conditioners whose initial VSOCs are uniformly distributed within $[0,1]$, switching functions are 0-1 integer distributed, and time intervals are 1 minute. The rated power is $2 \sim 3 \mathrm{~kW}$ uniformly distributed; the maximum charging time and discharge time are $10 \sim 15 \mathrm{~min}$ and $20 \sim 30 \mathrm{~min}$ uniformly distributed; the minimum temperature and maximum temperature of protocol are $24 \sim 25^{\circ} \mathrm{C}$ and $27 \sim 28^{\circ} \mathrm{C}$ uniformly distributed; the impact coefficient is $2 \sim 3$ uniformly distributed; and the starting current duration is $3 \sim 5$ s uniformly distributed. The maximum response potential was $128.25 \mathrm{~kW}$.

Assume that the shortage power is $30 \mathrm{~kW}$ and the protocol control period is 120 minutes, the related control results are shown in Figure 7.

Through reasonable dispatch during the protocol control period, the air conditioning load response power basically meets the shortage power, thus reducing the coincidence rate of the power system and making the power load operation more stable.

5.2. Analysis of Control Strategy Parameters of VSOC Priority Constant-Speed Air-Conditioners. Based on the example analyzed before, the influences of different power shortages and communication time intervals in the control strategy are discussed in this section. 


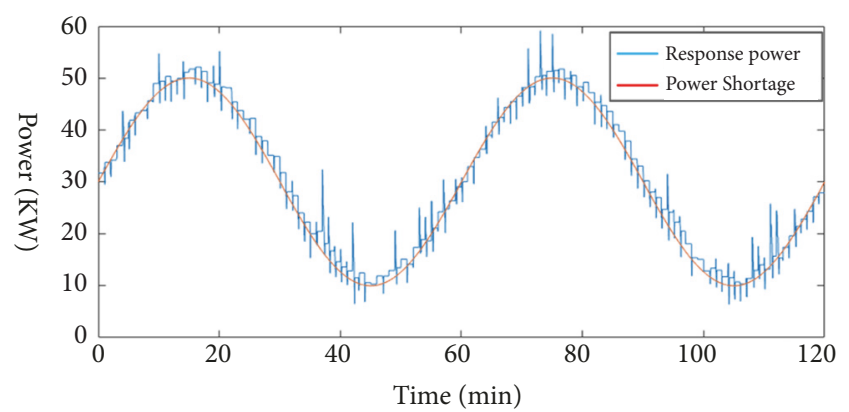

FIGURE 8: Tracking effect of VSOC priority constant-speed airconditioning control strategy.

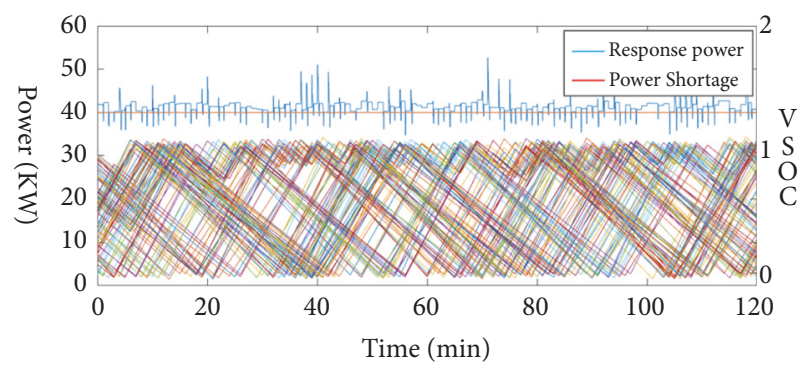

(a)

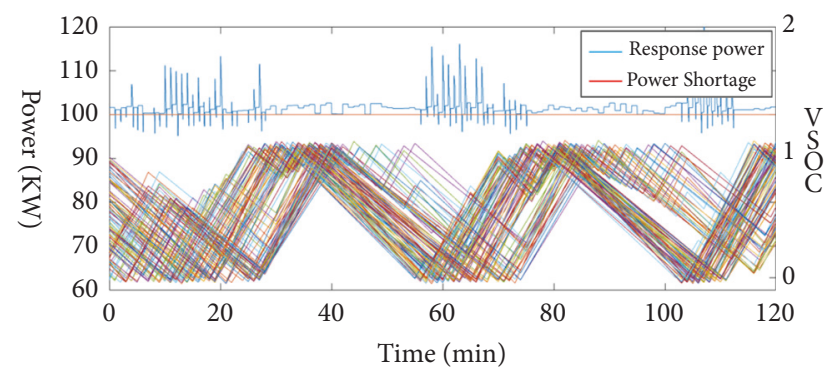

(b)

FIGURE 9: VES control effect of $40 \mathrm{~kW}$ and $100 \mathrm{~kW}$ power deficiency constant-speed air-conditioners.

5.2.1. Tracking Effect of VSOC Priority Constant-Speed AirConditioners Control Strategy. Assuming that the maximum power vacancy of demand-side response is $50 \mathrm{KW}$ and the minimum is $10 \mathrm{KW}$ sinusoidal curve, the control time is set to 120 minutes.

Figure 8 shows the tracking effect. As indicated in Figure 8 , the response power is in the capacity range, and the VSOC priority constant-speed air-conditioning control strategy can track the shortage power well. Power fluctuation and burr are mainly produced by the demand response of constant-speed air-conditioning.

5.2.2. Influence of Power Deviancy on Control Effect. $40 \mathrm{~kW}$, $100 \mathrm{~kW}, 130 \mathrm{~kW}$, and $150 \mathrm{~kW}$ are selected to analyze the control effect in the range of response power capacity and over the range of capacity. Results of control effect are shown in Figures 9 and 10.

When the gap power slightly exceeds the range, the initial response power will fluctuate massively, and the VSOC of its

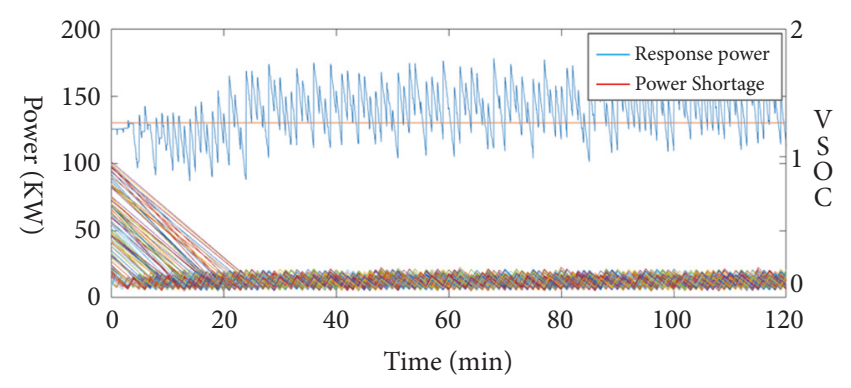

(a)

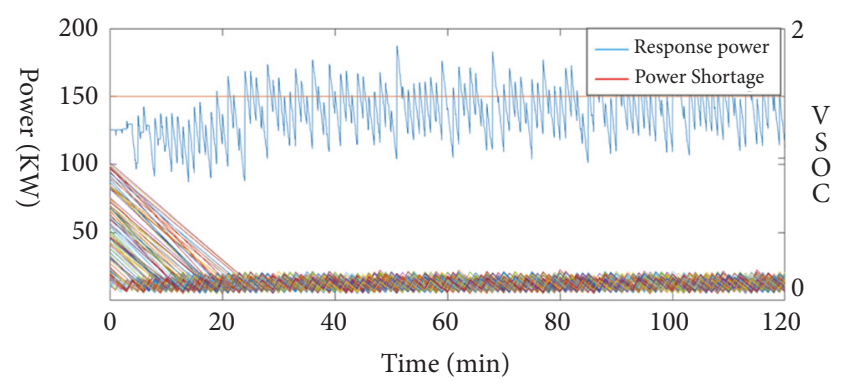

(b)

FIGURE 10: $130 \mathrm{~kW}$ and $150 \mathrm{~kW}$ power shortage constant-speed airconditioners VES control effect.

VES will oscillate at the bottom. After a period of adjustment, the response power can barely meet the power gap. With the gradual increase of the gap power, the response power will keep oscillating violently, and the VSOC state of VES will keep hovering at the bottom. As a result, the excessive response will lead to the violent oscillation of the response power. This will not only cause the failure to achieve response effect, but also pose greater threat by increasing the power oscillation in the distribution network.

5.2.3. Influence of Communication Time Interval on Control Effect. The communication time interval determines the refresh rate of the VES data, which will affect the control results. Figure 11 shows the control effect of VES in constantspeed air-conditioning with time interval of 2 minutes and 5 minutes, respectively. The whole process lasts 60 minutes. In order to achieve these results, the response power should be selected in the capacity range and the gap power of constantspeed air-conditioning should be set as $60 \mathrm{KW}$.

Communication time interval has a significant influence on VES of constant-speed air-conditioning. With the increasing of time interval, the constant-speed air-conditioning control strategy will gradually lose control over VES. The obvious power shortage will occur when the time interval is 5 minutes and the gap power is $60 \mathrm{KW}$. With the fluctuation range of power over $80 \%$, the maximum power vacancy can reach up to $50 \%$.

\section{Conclusion}

This paper proposed a VSOC prioritized virtual-energystorage control strategy based on the VES model of thermostatically controlled loads for constant-speed air-conditioner. 


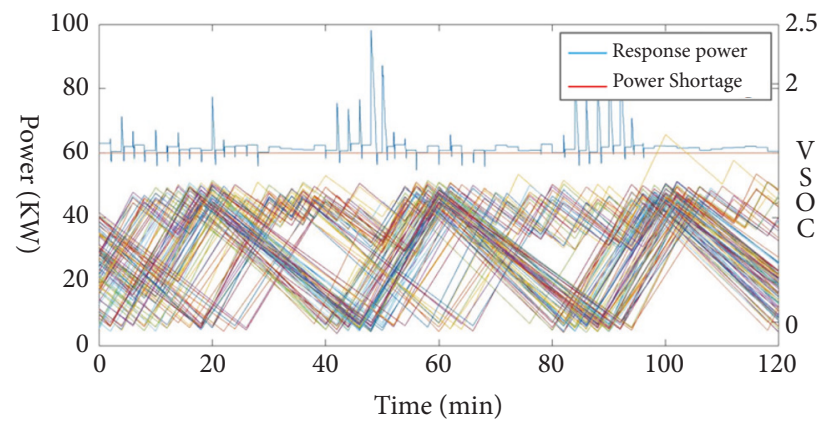

(a)

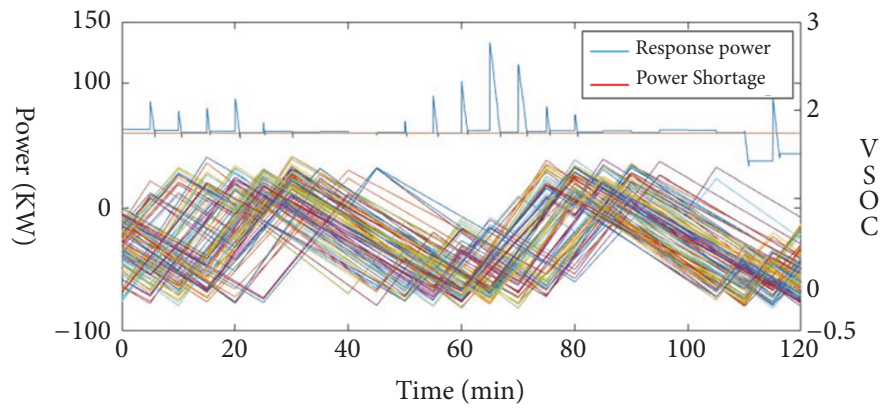

(b)

FIGURE 11: (a) and (b) show the control effect of VES in constant-speed air-conditioning with time intervals of 2 minutes and 5 minutes, respectively.

Relevant examples show that when the load resources of constant-speed air-conditioners effectively participate in the dispatch, the power shortage problem of the power system can be resolved and alleviated; consequently, the power efficiency of the system is significantly improved.

The simulation results prove the availability of VSOC prioritized virtual-energy-storage control model, which provides methodological support for demand-side of constantspeed air-conditioning loads to participate in power system operation. At the same time, it provides a control basis for other constant-speed compressor-typed TCL to participate in demand response. This is of great significance for the development of new energy absorption and intelligent power consumption, and it is expected to promote the progress of energy conservation and emission reduction.

The response power of VSOC prioritized constant-speed air-conditioner control strategy is limited by VES state and impact power duration. A stable power response can be achieved in a short time, but the control results in the long term still need further optimization. To overcome this shortcoming, it is the focus of future work studying the mechanism of the insufficient or excessive response of constant-speed compressor-typed TCLs in response process and optimizing the control effect of long-term VES.

\section{Data Availability}

The main program code data used to support the results of this study are included in the supplementary materials file (program code).

\section{Conflicts of Interest}

The authors declare that they have no conflicts of interest regarding the publication of this paper.

\section{Acknowledgments}

This project is supported by SGCC program: Research on Extensive Application and Benefit Evaluation of Typical Power Substitution Technology Considering Power Quality Influence $(52182018000 \mathrm{H})$ and Lanzhou Jiaotong UniversityTianjin University Innovation Fund Program: Research on
Management Strategies of Power Quality Problems from Thermostatically Controlled Appliances (2018058).

\section{Supplementary Materials}

The Supplementary Materials file includes the process of modeling process of virtual energy storage model. The main idea of ETP modeling method is to equate the cooling (heat) of the room (box) and the external environment as well as the energy conversion with the related circuit components such as resistors, capacitors, and power supply. The relationship between temperature and energy conversion is analyzed by circuit knowledge. Second-order thermodynamic model is widely used. When the temperature changes relatively slowly, there is no obvious difference between gas and solid temperatures. In order to increase the practicability of the model, the second-order ETP model can be reduced to the first-order ETP model if the temperature of gas and solid in the room is equal. On the basis of ETP model, the virtual energy storage model of temperature-controlled load is established, taking fully into account the characteristics of energy and heat exchange. Virtual energy storage can be divided into thermal and electric parts. See the article for a detailed introduction of the thermal and electric parts. This is the modeling process of virtual energy storage model. (Supplementary Materials)

\section{References}

[1] H. Zhao, J. Zhou, and E. Yu, "Advanced metering infrastructure supporting effective demand response," Dianwang Jishu/Power System Technology, vol. 34, no. 9, pp. 13-20, 2010.

[2] S. Yuanwei, L. Yang, G. Zhiwei et al., "Application of demand response in ancillary service market," Power System Automation, vol. 22, pp. 157-167, 2017.

[3] L. Huiping, "Internet + intelligent energy' action helps more beautiful life," Shanghai Energy Saving, vol. 11, pp. 6-12, 2017.

[4] F. Qingdong and H. Zhanyong, "Tactics of direct load control in demand response," Electrical Measurement and Instruments, vol. 49, no. 3, pp. 59-63, 2012.

[5] J. H. Kämpf and D. Robinson, "A simplified thermal model to support analysis of urban resource flows," Energy and Buildings, vol. 39, no. 4, pp. 445-453, 2007. 
[6] A. Pahwa and C. W. Brice, "Modeling and system identification of residential air conditioning load," IEEE Transactions on Power Apparatus and Systems, vol. 104, no. 6, pp. 1418-1425, 1985.

[7] J. Peppanen, M. J. Reno, and S. Grijalva, "Thermal energy storage for air conditioning as an enabler of residential demand response," in Proceedings of the North American Power Symposium, NAPS '14, pp. 1-6, IEEE, 2014.

[8] N. Lu and D. P. Chassin, "A state-queueing model of thermostatically controlled appliances," IEEE Transactions on Power Systems, vol. 19, no. 3, pp. 1666-1673, 2004.

[9] C. F. Walker and J. L. Pokoski, "Residential load shape modeling based on customer behavior," IEEE Transactions on Power Apparatus and Systems, vol. 104, no. 7, pp. 1703-1711, 1985.

[10] F. Luo, Z. Y. Dong, K. Meng, J. Wen, H. Wang, and J. Zhao, “An operational planning framework for large-scale thermostatically controlled load dispatch," IEEE Transactions on Industrial Informatics, vol. 13, no. 1, pp. 217-227, 2017.

[11] X. Ai, Y. Zhao, and S. Zhou, "Study on virtual energy storage features of air conditioning load direct load control," Zhongguo Dianji Gongcheng Xuebao/Proceedings of the Chinese Society of Electrical Engineering, vol. 36, no. 6, pp. 1596-1603, 2016.

[12] D. Yuxin, L. Wang, L. Yang et al., "Direct load control strategies and optimization scheduling of thermostatically controlled loads," Journal of Power Systems and Automation, vol. 27, no. 6, 2015.

[13] L. Mei, Y. Bo, and Y. Kemin, "Research status and application prospects of human comfort," Meteorological Science and Technology, vol. 30, no. 1, pp. 11-14, 2002.

[14] C. Bin, Research on the Impacts of Climate and Built Environment on Human Thermal, Tsinghua University, 2012.

[15] T. Yibin, Y. Xiaojie, W. Yilan et al., "Research on virtual energy storage of air conditioning load," Beijing Jiaotong Daxue Xuebao/Journal of Beijing Jiaotong University, vol. 41, no. 5, pp. 126-131, 2017.

[16] D. Wang, F. Menghua, and J. Hongjie, "User comfort constraint demand response for residential thermostatically-controlled loads and efficient power plant modeling," Zhongguo Dianji Gongcheng Xuebao/Proceedings of the Chinese Society of Electrical Engineering, vol. 34, no. 13, pp. 2071-2077, 2014.

[17] X. Siyuan, C. Yanbo, H. Wei et al., "Control strategy of electric heating loads for reducing power shortage in power grid," Processes, vol. 7, no. 5, article 273, 2019.

[18] C. Gao, Q. Li, and Y. Li, "Bi-level optimal dispatch and control strategy for air-conditioning load based on direct load control," Zhongguo Dianji Gongcheng Xuebao/Proceedings of the Chinese Society of Electrical Engineering, vol. 34, no. 10, pp. 1546-1555, 2014.

[19] L. Zhou, Y. Li, and C. Gao, "Improvement of temperature adjusting method for aggregated air-conditioning loads and its control strategy," Zhongguo Dianji Gongcheng Xuebao/Proceedings of the Chinese Society of Electrical Engineering, vol. 34, no. 31, pp. 5579-5589, 2014.

[20] C. Li, Q. Yang, Y. Wei et al., "Distribution network reconfiguration considering correlationbetween wind-speed and load," Power automation equipment, vol. 36, no. 2, pp. 148-153, 2016.

[21] Z. Qiqi, O. Fan, G. Qiang et al., "Load characteristic comparison between inverter and constant-speedair conditioner and their influences on power grid," East China Electric Power, vol. 42, no. 10, pp. 2017-2021, 2014.

[22] Y. Dan, Optimal Dispatch of Load in Smart Grids based on Resident Behavour, Shenyang University, 2013.
[23] Federal Energy Regulatory Commission, "Assessment of demand response and advanced metering," Staff Report [EB/OL], 2006.

[24] N. Lu, "An evaluation of the HVAC load potential for providing load balancing service," IEEE Transactions on Smart Grid, vol. 3, no. 3, pp. 1263-1270, 2012. 


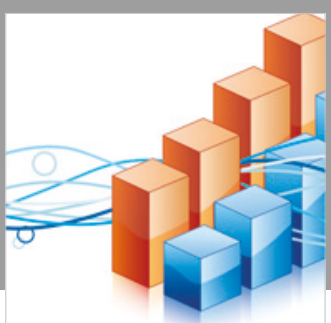

Advances in

Operations Research

\section{-n-m}
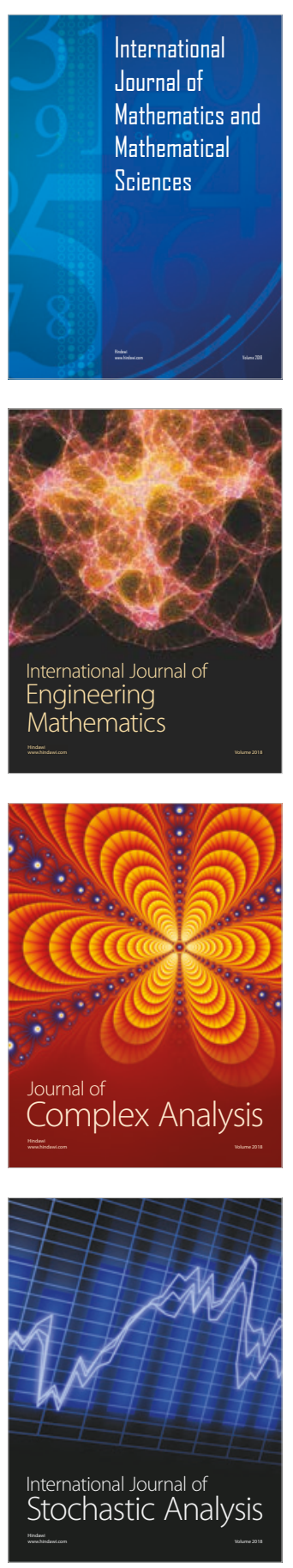
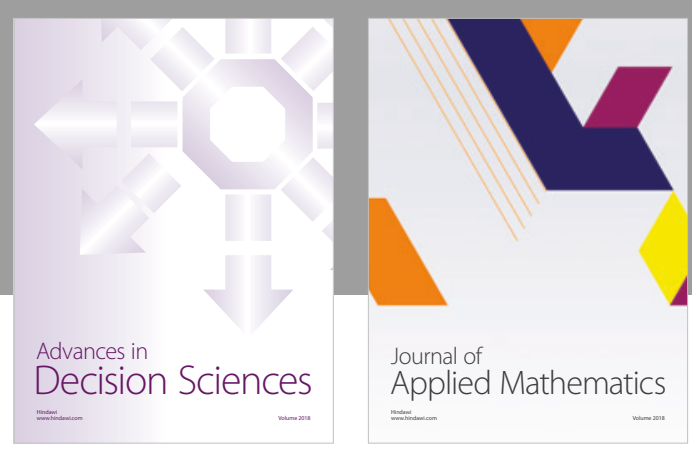

Journal of

Applied Mathematics
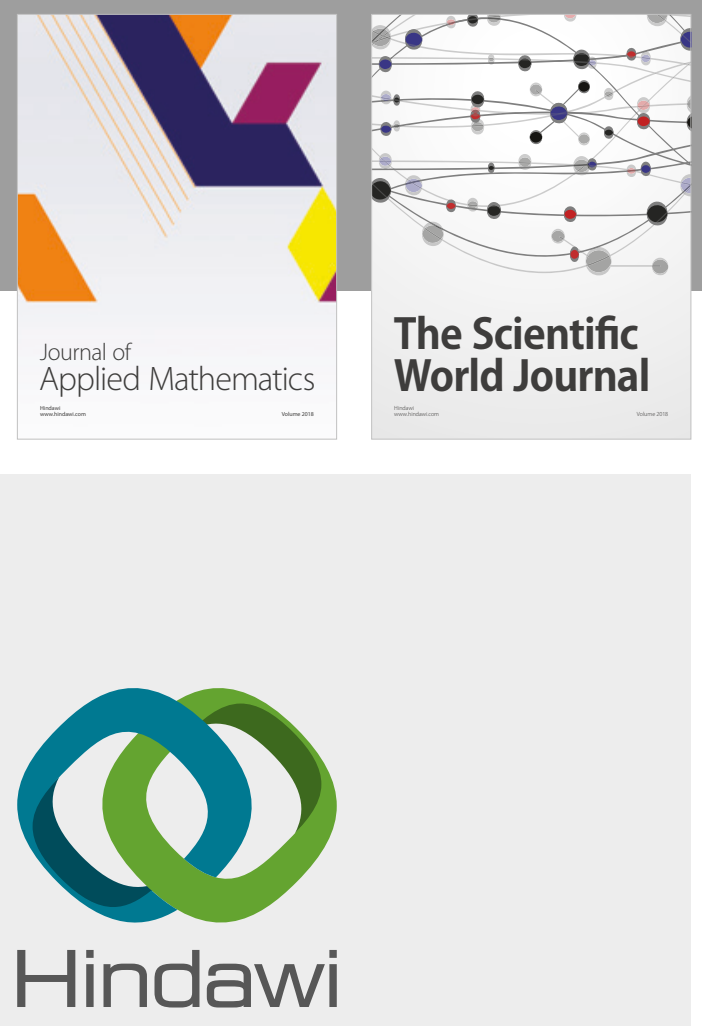

Submit your manuscripts at

www.hindawi.com

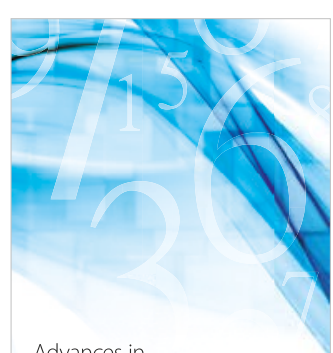

Advances in
Numerical Analysis
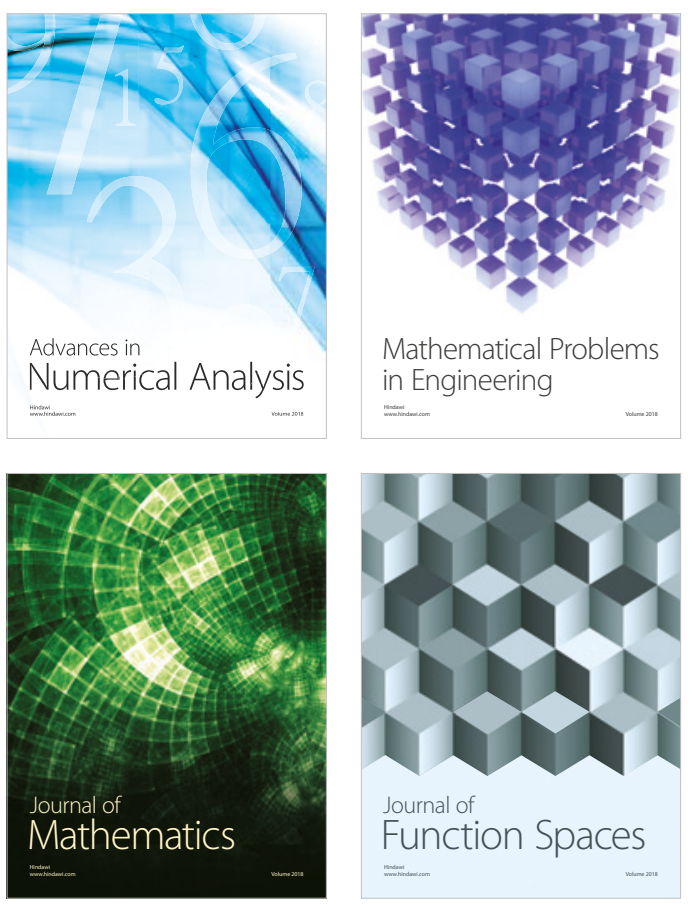

Mathematical Problems in Engineering

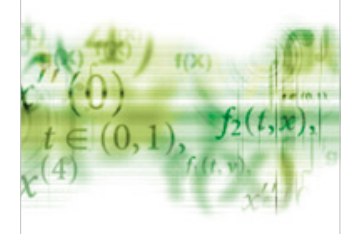

International Journal of

Differential Equations

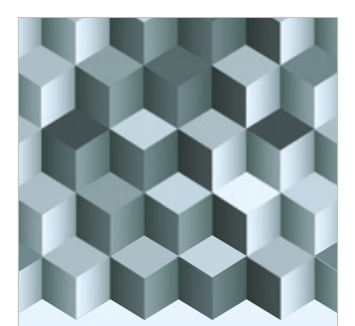

Journal of

Function Spaces
The Scientific

World Journal

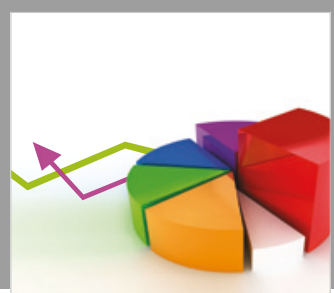

Journal of

Probability and Statistics
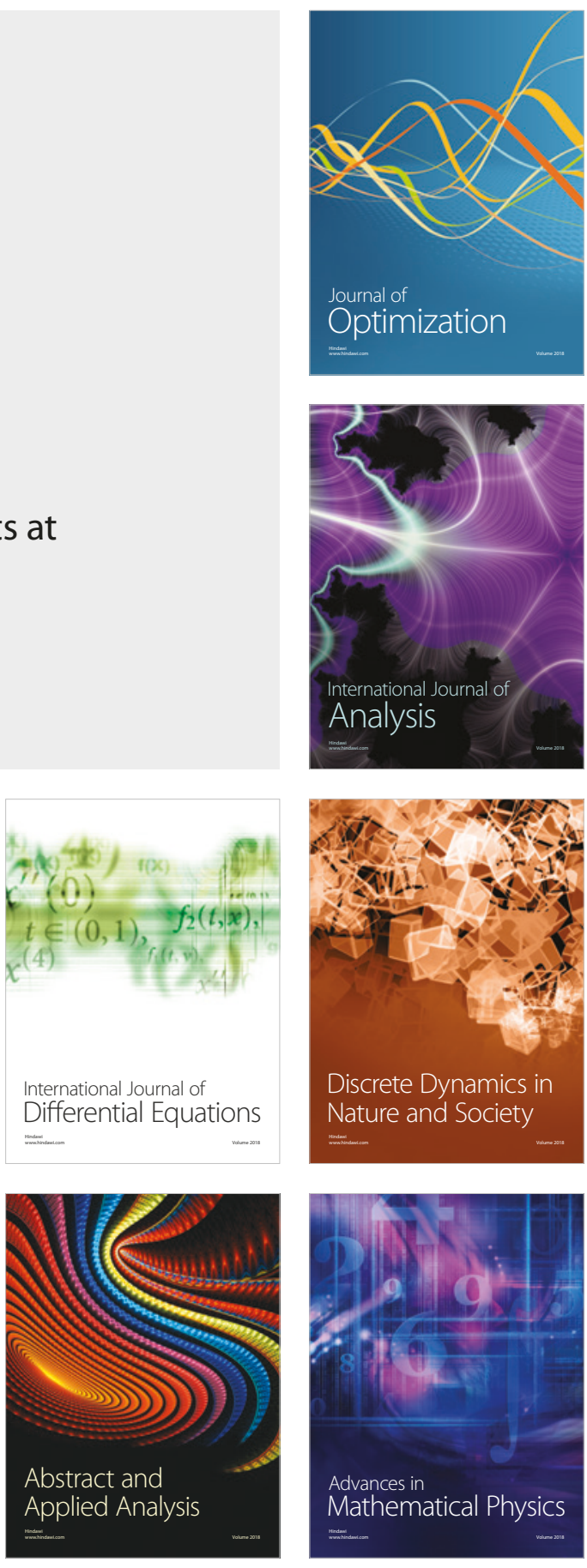